\title{
Spectrophotometric detection of susceptibility to anti-malarial drugs
}

\author{
Yulia M Serebrennikova ${ }^{1,2^{*}}$, Janus Patel ${ }^{1}$, Wilbur K Milhous ${ }^{1}$, Luis H Garcia-Rubio², Debra E Huffman² \\ and Jennifer M Smith ${ }^{2}$
}

\begin{abstract}
Background: With malaria drug resistance increasing in prevalence and severity, new technologies are needed to aid and improve the accuracy and clinical relevance of laboratory or field testing for malaria drug resistance. This study presents a method based on simple and reagentless spectroscopic measurements coupled with comprehensive spectral interpretation analysis that provides valuable quantitative information on the morphological and compositional responses of Plasmodium falciparum and infected red blood cells (IRBCs) to anti-malarial treatment.
\end{abstract}

Methods: The changes in the size, internal structure, nucleotide and haemozoin composition of the parasites as well as the morphology (size and shape) and haemoglobin composition of the IRBCs treated with dihydroartemisinin (DHA) and mefloquine (MFQ) were investigated using a spectral interpretation analysis.

Results: DHA treatment reduced the sizes of the parasites and their structural organelles. The haemoglobin composition of the host IRBCs determined from spectroscopic analysis changed negligibly following DHA treatment. MFQ treated parasites grew to the same size as those from parallel non-treated cultures but lacked haemozoin. Lesser deformation of the cell shape and no haemoglobin depletion were detected for the IRBCs of MFQ treated cultures.

Conclusions: The spectroscopic analysis method proved to be sensitive for recognition of the effects of anti-malarial treatment on the structure and composition of the parasites and IRBCs. The method can have significant potential for research and clinical applications such as evaluating patient specimens for drug action, drug effects or for therapeutic monitoring.

Keywords: Malaria, Antimalarial, Spectroscopy, Spectral interpretation

\section{Background}

Microscopy has long been the "gold standard" as a tool for the research based characterization of malaria infected red blood cells (IRBCs) and for the detection of malaria parasites in the field [1-5]. Because this technique is constrained by substantial technical and economic hurdles such as operator training, time needed to perform a test, and labor intensiveness, there is an ongoing search for alternatives. Immunochromatography-based rapid diagnostic tests have gained widespread popularity because of ease-of-use but often suffer from high variability, poor

\footnotetext{
*Correspondence: yulia.serebrennikova@clarosci.com

${ }^{1}$ College of Public Health, University of South Florida, 13201 Bruce B. Downs Blvd., Tampa, FL 33612, USA

${ }^{2}$ Claro Scientific 10100 Dr, Martin Luther King Jr. St. N., St., Petersburg, FL 33716, USA
}

\section{Biomed Central}

sensitivity for mixed infections of Plasmodium species other than Plasmodium falciparum, extensive requirements for reagent quality control $[2,3,6]$. Techniques based on polymerase chain reaction (PCR) offer superior sensitivity, ability to detect mixed species infection and the ability to differentiate among four species of Plasmodium but they require considerable cost of equipment as well as storage and maintenance requirements for reagents $[2,4]$. Quantitative PCR has been repeatedly demonstrated to be more accurate than microscopy for the detection of malaria at very low levels of parasitaemia $[7,8]$ and it disagrees with microscopy for parasitaemia counts at higher parasitaemia levels [8]. It is not straightforward and requires complicated analysis to assess the sensitivity of the malaria parasites to anti-malarials using immunochromatography or molecular 
methods [9-11] and is time-consuming when using classical in vivo studies [12].

UV-visible spectroscopy that explores spectral changes in the infected red blood cells is a new tool in malaria diagnostics that overcome most of the disadvantages of the current malaria diagnostics methods by being rapid, sensitive and quantitative. Spectroscopic and light scattering techniques have been a subject of continuous interest since these non-destructive measurements provide substantial information on the physical, chemical, and physiological character of cells and, therefore, can potentially detect and identify changes in cells that are indicative of diseases. For instance, flow cytometry and commercial haematology analyzers utilizing abnormalities in the multiple-angle polarized scattering plots have demonstrated potential for the detection of malaria parasites in blood with reported sensitivities of $\geq 95 \%$ in samples with $>100$ parasites/ $\mu \mathrm{l}$ and is in compliance with WHO malaria-diagnostic guidelines $[1,13]$.

Given that the asexual stages of the malaria parasites occur inside red blood cells (RBCs), assessment of the changes in the red blood cell (RBC) properties is a valuable approach for infection detection and characterization. This prominent component of blood has long been attractive to the spectroscopic investigations as a light scatterer with a homogeneous body and the distinct spectroscopic features of its main constituent, haemoglobin. The IRBC morphology and composition appreciably affect the spectra with the progression of the intraerythrocytic development of malaria parasites. Even though the size and shape of an IRBC at the ring stage remain the same as those of noninfected RBCs [14], the parasite with its parasitophorus vacuole occupies $5-15 \%$ of the host volume [15-17]. The parasite occupies about one third of the host IRBC by the trophozoite stage $[16,18]$ and more than a half of the host volume through the subsequent schizont stage $[15,18,19]$. This growth is accompanied with the loss of shape, swelling, and formation of protrusions on the IRBC surface $[18,20,21]$. The parasite continuously uptakes haemoglobin from the host cytosol and converts it into haemozoin deposited into the parasite's digestive vacuole [22-24]. Haemoglobin depletion of the host and haemozoin build up in the parasite's vacuole progress with the parasite's development and become the greatest at the schizont stage [22-24]. It has been demonstrated for different intraerythrocytic life stages of $P$. falciparum that the UV-visible spectroscopy can track these changes [25].

Since anti-malarial treatment leads to the morphological and/or compositional changes in the parasites, in this study it was hypothesized that anti-malarial effect can be captured by UV-visible measurements. The objectives of this study were to examine the hypothesis with the experimentally acquired data using dihydroartemisinin (DHA) and mefloquine (MFQ), to effectively obtain quantitative measurements of the morphological parameters, haemoglobin composition of the IRBCs and composition of the parasites following the treatment, and to show the sensitivity of the method for the recognition of the effects of anti-malarial treatment. To achieve the objectives, simultaneous measurements of the absorption and forward scattering in the UV-visible-NIR portion of the electromagnetic spectrum were coupled with a spectral interpretation model based on an ellipsoidal approximation to the multilayered Mie theory. The parameters such as the size, nucleotide and haemozoin composition of the parasites as well as the size, shape and haemoglobin composition of the IRBCs could serve as the determining factors of the susceptibility or resistance of the parasite.

\section{Methods}

\section{Sample preparation}

In vitro cultures of the W2 strain P. falciparum were grown at $4 \%$ hematocrit in RPMI media to $6-14 \%$ parasitaemia following the standard method [26]. Cultures were synchronized with both $\mathrm{D}$-sorbitol and incubation temperature cycling of $17^{\circ} \mathrm{C}$ and $40^{\circ} \mathrm{C}$ to achieve a high concentration of ring stage parasites. Five independent experiments were conducted. In each experiment, cultures were prepared by either splitting a parent culture into subcultures, or growth of two or three parallel cultures. One subculture or parallel culture was set as a control (no drug exposure) and others were treated with either mefloquine (MFQ) or dihydroartemisinin (DHA). The exposure concentration of each drug was $1,500 \mathrm{ng} / \mathrm{mL}$ (7.5 $\mu \mathrm{l}$ of $1 \mathrm{mg} / \mathrm{ml} \mathrm{MFQ}$ or DHA solution was added to a $5 \mathrm{ml}$ culture). The experiments always started with all cultures being at the ring stage. The parasite extraction from IRBCs was performed by saponin lysis as previously described [14]. Briefly, $1 \mathrm{ml}$ of $0.1 \%$ saponin solution in PBS was added to $0.5 \mathrm{ml}$ of approximately $50 \%$ haematocrit $\mathrm{RBC}$ suspension. The mixture was incubated at $37^{\circ} \mathrm{C}$ with constant agitation for 15 minutes. Then, it was centrifuged at 13,000 rpm for $1 \mathrm{~min}$ and the pellet was resuspended in PBS. The procedure was repeated 3-4 times to remove the dissolved haemoglobin and the RBC membrane fragments. Visible microscopy inspection was performed to confirm the integrity of the extracted parasites.

\section{Spectrophotometric measurements}

To measure the UV-visible-NIR spectra of IRBC cultures, $0.5 \mathrm{ml}$ of each culture was centrifuged at 13,000 rpm for 30 seconds, growth media was aspired, and the cells were suspended in $1 \mathrm{ml}$ of phosphate buffered saline (PBS). The procedure was repeated 3-4 times until the supernatant was clear of the growth media. The UV-visible-NIR spectra were recorded using a diode array spectrometer (Agilent 8453 Santa Clara, CA) having an acceptance angle smaller than $2^{\circ}$ at room temperature using a $1 \mathrm{~cm}$ 
pathlength cuvette. The measurements were conducted with an acquisition time of $0.5 \mathrm{sec}$, signal to noise ratio greater than 1000, and $1 \mathrm{~nm}$ wavelength resolution. Prior to recording a sample spectrum the spectrometer was zeroed to account for any stray light. The background spectrum was taken using a sample from the same PBS batch utilized in the preparation of the RBCs suspensions. Twenty microlitres of prepared RBC suspension was added to $2 \mathrm{ml}$ of PBS in a quartz cuvette and inverted to ensure homogeneous distribution of RBCs in the cuvette. For each culture sample, 5-8 replicate spectral measurements were taken. The spectral measurements were taken at 10-11, $15-17$, and 22 (for MFQ treated and control cultures) hrs post treatment.

\section{Spectral interpretation analysis}

The structure of the spectral interpretation model for IRBCs was described in detail in Serebrennikova et al. [25]. In brief, the model included a three-layer Mie geometry (i.e., a sphere having three concentric layers) to account for changes in the refractive indices of the IRBC cytosol and the parasite's cytoplasm and organelles. The model incorporated an ellipsoidal approximation to Mie theory to account for the effects of the RBC orientation and non-spherical shape. It has been recently demonstrated that this approximation can accurately predict the spectral features of oriented composite ellipsoids [27]. The ellipsoidal approximation to the RBC shape is quantitatively suitable for the prediction of light attenuation by RBCs in the forward direction [28]. According to the model the measured optical density was theoretically predicted as a weighted sum of the extinctions of orientation populations:

$$
\tau(\lambda)_{\text {calc }}=N_{p} L \sum \omega_{i}\left(A_{i} / 4\right) Q_{e x t, i}
$$

where $\tau(\lambda)_{\text {calc }}$ is the predicted total optical density at given wavelength $\lambda, \mathrm{N}_{\mathrm{p}}$ is the total number density of cells, $\mathrm{L}$ is the pathlength, $\omega_{1}$ is the weight fraction of the $i^{\text {th }}$ orientation, $A_{i}$ and $Q_{\text {ext }, i}$ are the projected area and extinction, respectively, of the cells at the $i^{\text {th }}$ orientation. The extinction $Q_{\text {ext }}$ of each IRBC orientation was, in turn, computed as weighted sum of the extinctions of three structural groups (Figure 1):

$$
Q_{e x t, i}=\omega_{D V} Q_{e x t, D V}+\omega_{N U} Q_{e x t, N U}+\omega_{O R G} Q_{e x t, O R G}
$$

Each structural group was modelled as a three-layer structure such that the outer layer was the IRBC cytosol, the intermediate layer was the parasite's cytoplasm, and the core was a parasite's structural element. Three structural elements were suggested by the available information on the morphological structure of $P$. falciparum cells [15]. Those were digestive vacuole (DV) containing haemozoin, nucleus (NU) containing nucleic acid that provides distinct spectral contribution, and the third structural element was the population of other protozoan organelles. This "average" organelle (ORG) was modelled using the average refractive index of non-chromophoric molecules of biological cells [29]. Validated approximation of additivity of the spectral contributions from the structural groups $[25,29]$ was used. The extinction of each structural group was a function of the size parameters and refractive indices of the layers. The size parameter of each layer $(\mathrm{X})$ was defined as:

$$
X=2 \pi n_{0} D_{M I E} / \lambda
$$

where $n_{O}$ is the refractive index of the medium and $D_{M I E}$ is the diameter. In the case of the outer layer (i.e., RBC cytosol) $D_{\text {MIE }}$ denotes the equivalent sphere diameter of the ellipsoid computed according to [30] as:

$$
\begin{aligned}
& D_{M I E}=a / g \\
& g=\left(\cos ^{2} \psi+\left(\cos ^{2} \xi+r^{2} \sin ^{2} \xi\right) \sin ^{2} \psi\right)^{1 / 2}
\end{aligned}
$$

where $r=a / c$ is the ratio of the symmetry axis $a$ to minor axis $c$ of the ellipsoid and $\psi$ and $\xi$ are the rotation angles of the ellipsoid's $x-z$ and $y-z$ planes with respect to the direction of the incident beam. The real $n$ and imaginary $k$ parts of the complex refractive index of the $l^{\text {th }}$ layer were computed as weighted sums of the refractive indices of its compositional constituents:

$$
\begin{aligned}
& k_{l}=\Sigma v_{l j} k_{j} \\
& n_{l}=\Sigma v_{l j} n_{j}
\end{aligned}
$$

where $v_{i j}$ is the weight fraction of the $j^{\text {th }}$ compositional constituent in the $t^{\text {th }}$ layer (Table 1). The outer layer was modelled to be composed of haemoglobin and water. The intermediate layer (i.e. parasite cytoplasm) was modelled to be composed of water and non-absorbing macromolecules [29]. The core elements were modelled as following: nucleus was composed of nucleic acid, non-absorbing macromolecules, and water; digestive vacuole was composed of water and haemozoin [25]; and the "average" organelle was composed of water and non-absorbing macromolecules. The refractive index of nucleic acid was constructed from those of the purine and pyrimidine bases [29] assuming $\mathrm{G}+\mathrm{C}$ fraction of 0.2 for P. falciparum [31].

The interpretation procedure consisted of the prediction of the measured spectra as functions of the model parameters and subsequent comparison of the predicted and measured spectra using an iterative least squares 


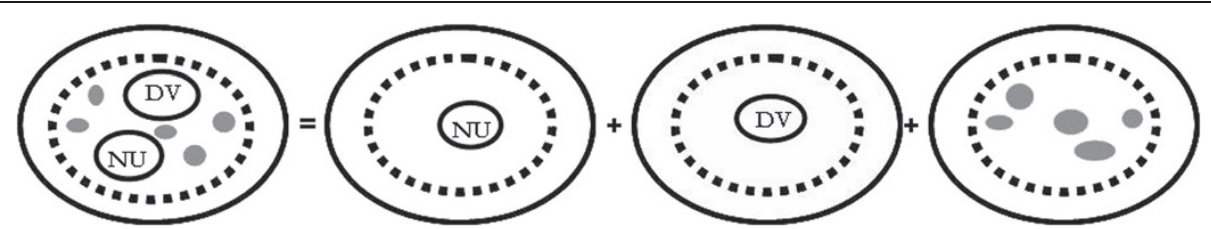

Figure 1 Schematics of the $P$. falciparum infected RBC model. IRBC was approximated as a weighted sum of three structural groups each having three concentric layers. The outer layer was IRBC cytosol, the middle layer (dashed line) was the parasite's cytoplasm and the core was either digestive vacuole (DV), nucleus (NU), or organelles (ORG).

minimization procedure. The residual sum of squares (RSSQ) was calculated as:

$$
R S S Q=(1 / M) \Sigma\left(\tau(\lambda)_{\text {meas }, m}-\tau(\lambda)_{c a l c, m}\right)^{2}
$$

where $M=651$ and corresponds to the $250-900 \mathrm{~nm}$ wavelength range and $1 \mathrm{~nm}$ wavelength resolution. Model parameters were iterated until convergence (i.e., relative changes in RSSQ were less than $10^{-5}$ ) using a Nelder-Meade downhill simplex optimization algorithm and variable transformation techniques $[14,25]$.

The outputs of the interpretation analysis were used to reconstruct the measured spectra of IRBCs. In order to eliminate the orientation effect [25] and, therefore, to directly compare the effects of the structural and compositional differences, the IRBC spectra were reconstructed with the same orientation parameters. The interpretation of the measured UV-visible-NIR spectra of $P$. falciparum cells extracted from IRBCs was conducted using two-layer Mie theory as described in detail in [14].

\section{Results and discussion}

\section{Untreated parasites}

The average reconstructed spectra of non-infected healthy RBCs (H), control IRBCs (IC), MFQ treated IRBCs (IM), and DHA treated IRBCs (ID) at 10-11 hrs post treatment are contrasted in Figure 2. Significant differences in the spectral features could be noted. Comparison of

Table 1 The composition of the structural groups of the IRBC interpretation model

\begin{tabular}{cccc}
\hline $\begin{array}{c}\text { Structural group } \\
\text { (shell/core): }\end{array}$ & $\begin{array}{c}\text { Composition } \\
\text { of the shell }\end{array}$ & $\begin{array}{c}\text { Composition } \\
\text { of the shell }\end{array}$ & $\begin{array}{c}\text { Composition } \\
\text { of the core }\end{array}$ \\
\hline IRBC body/ & & Haemozoin \\
Parasite body/ & Haemoglobin & Water \\
$\begin{array}{c}\text { Digestive vacuole } \\
\text { Haemoglobin }\end{array}$ & Proteins & Proteins \\
Parasite body/ & Water & RNA & DNA + RNA + \\
nucleotides & Water \\
Nucleus & & Water & Proteins \\
IRBC body/ & & Water \\
Parasite body/ & & \\
Organelles & & \\
\hline
\end{tabular}

non-infected RBCs and IRBC controls revealed spectral changes that were due to the structural and compositional alterations to IRBCs caused by intraerythrocytic development of parasites. The interpretation models allowed the calculation of parameters relating to the morphology and haemoglobin composition of IRBCs as well as the size, structure, nucleotide and haemozoin composition of parasites from the measured spectra of IRBCs and extracted parasites. These structural and compositional parameters of the parasites in control cultures are given in Table 2. Figure 3 compares their measured and reconstructed spectra as well as the spectral contributions of the modelled structural groups. The structural and compositional parameters of IRBC control cultures are given in Table 3.

Comparison between the parameters of control parasites obtained at 10-11 and 15-22 hrs revealed progressive changes in the sizes of the cells and organelles as well as haemozoin and nucleotide content of the cells indicating growth and metabolic activity. For instance, the estimated cell volume for $P$. falciparum increased from $16 \mu \mathrm{m}^{3}$ at $10-11 \mathrm{hrs}$ to $20-22 \mu \mathrm{m}^{3}$ at $15-22 \mathrm{hrs}$. These values showed a transition from the ring to trophozoite stages paralleling other reports of cell volumes $\left(10 \pm 5 \mu \mathrm{m}^{3}\right.$ and $\left.30 \pm 10 \mu \mathrm{m}^{3}\right)$ for the ring and trophozoite stages, respectively $[15,17]$. The digestive vacuole volume has been reported to range from $4 \mu \mathrm{m}^{3}$ at the early trophozoite stage $[15,24]$ to $9-11 \mu \mathrm{m}^{3}$ at later developmental stages [20]. The DV volumes estimated in this study were in good agreement with published data and also suggested early trophozoite stage at 10-11 hrs and more advanced mid-trophozoite stage at 15-22 hrs for the

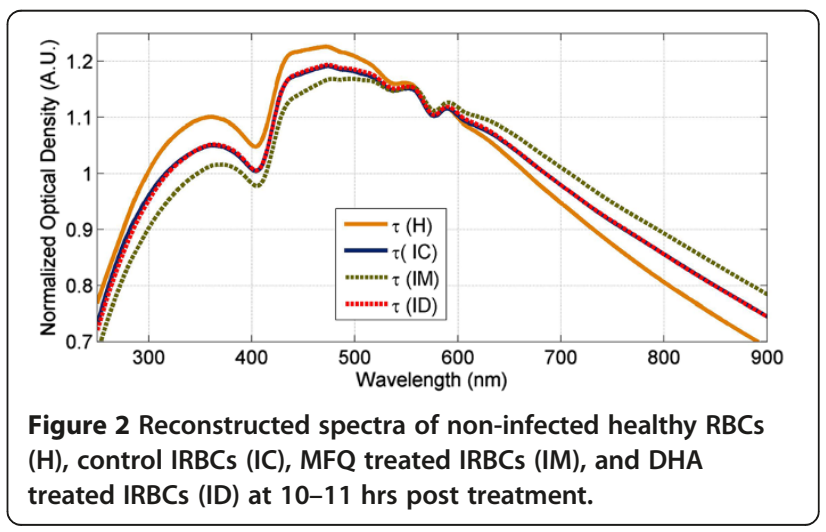


Table 2 Structural and compositional parameters of $P$. falciparum obtained through interpretation of the measured UV-visible-NIR spectra of control IRBCs and parasites extracted from the host IRBCs

\begin{tabular}{llll}
\hline Paramer & $\mathbf{1 0 - 1 1} \mathbf{~ h r s}$ & $\mathbf{1 5 - 1 7} \mathbf{~ h r s}$ & $\mathbf{2 2} \mathbf{~ h r s}$ \\
\hline Average cell volume (fl) & $16.0 \pm 0.7$ & $20.7 \pm 0.4$ & $22.4 \pm 1.9$ \\
DV volume (fl) & $3.9 \pm 0.2$ & $5.4 \pm 0.2$ & $6.0 \pm 0.2$ \\
NU volume (fl) & $2.8 \pm 0.4$ & $2.7 \pm 0.5$ & $2.8 \pm 0.3$ \\
HZ (pg/cell) & $0.15 \pm 0.03$ & $0.28 \pm 0.04$ & $0.27 \pm 0.01$ \\
Nucleotides (fg/cell) & $45 \pm 12$ & $141 \pm 30$ & $120 \pm 21$ \\
\hline
\end{tabular}

controls. Published micrographs of $P$. falciparum suggest that the nucleus occupies about $10-25 \%$ of the cell volume [15] and are in agreement with nucleus volumes obtained in this study $\left(2.2-2.8 \mu \mathrm{m}^{3}\right.$ ) (Table 2). The amount of total nucleotide in control parasites (Table 2) was in agreement with our previous estimates made for $P$. falciparum trophozoite stage [14]. The amounts of haemozoin were $0.15 \mathrm{pg} / \mathrm{cell}$ and $0.27-0.3 \mathrm{pg} / \mathrm{cell}$ at $10-11 \mathrm{hrs}$ and 15-22 hrs, respectively, for the controls. These values also corresponded well to the early and and mid-trophozoite stages of $P$. falciparum [23,24].

Even though control IRBCs retained the same volume as non-infected RBCs at 10-11 hrs, the cell aspect ratio (i.e., the ratio of the width to length) increased indicating changes in the cell shape (Table 3). The haemoglobin content of control IRBCs was also reduced due to its uptake by the parasites. The volume and aspect ratio of control IRBCs continued to increase whereas haemoglobin content continued to decrease from the 10-11 to 15-17 and to $22 \mathrm{hrs}$ measurements in response to the intraerythrocytic growth of $P$. falciparum (Table 3). The
Table 3 Structural and compositional parameters of non-infected healthy RBCs and control IRBCs obtained through interpretation of measured UV-visible-NIR spectra

\begin{tabular}{|c|c|c|c|c|}
\hline \multirow[t]{2}{*}{ Parameter } & \multirow{2}{*}{$\begin{array}{l}\text { Non-infected } \\
\text { RBCs }\end{array}$} & \multicolumn{3}{|c|}{ IRBCs } \\
\hline & & 10-11 hrs & $15-17 \mathrm{hrs}$ & $22 \mathrm{hrs}$ \\
\hline RBC volume $\left(\mu \mathrm{m}^{3}\right)$ & $83.1 \pm 0.7$ & $85.6 \pm 1.0$ & $87.8 \pm 1.4$ & $99.6 \pm 1.8$ \\
\hline RBC length ( $\mu \mathrm{m})$ & $8.45 \pm 0.03$ & $8.45 \pm 0.03$ & $8.26 \pm 0.05$ & $7.95 \pm 0.06$ \\
\hline RBC width $(\mu \mathrm{m})$ & $2.22 \pm 0.02$ & $2.29 \pm 0.02$ & $2.47 \pm 0.06$ & $2.98 \pm 0.08$ \\
\hline $\mathrm{MCHC}\left(\mathrm{g} \mathrm{ml}^{-1}\right)$ & $30.4 \pm 0.5$ & $32.0 \pm 0.0$ & $28.3 \pm 0.6$ & $24.0 \pm 0.1$ \\
\hline $\mathrm{MCH}\left(\mathrm{pg} \mathrm{cell}^{-1}\right)$ & $25.5 \pm 0.6$ & $21.6 \pm 1.0$ & $20.0 \pm 0.7$ & $18.2 \pm 0.5$ \\
\hline$M C H_{1}\left(p g\right.$ cell $\left.^{-1}\right)$ & - & $25.7 \pm 0.6$ & $25.1 \pm 0.6$ & $25.7 \pm 0.7$ \\
\hline
\end{tabular}

MCHC mean corpuscular haemoglobin concentration, $\mathrm{MCH}$ mean corpuscular haemoglobin amount per cell, $M C H C_{1}$ estimated initial $M C H$ of IRBCs.

changes in the aspect ratio indicated swelling of IRBCs; IRBC swelling has been previously demonstrated to occur in response to the parasite growth through trophozoite stage $[20,32,33]$.

Haemoglobin losses from the IRBC cytosol to the parasite were $15 \%$ at $10-11 \mathrm{hrs}, 20 \%$ at $15-17 \mathrm{hrs}$, and up to $30 \%$ at 22 hrs. These values were in agreement with those from other studies utilizing other approaches such as refractive index mapping and colorimetric iron determination. These reports indicate $5-10 \%$ and $40-60 \%$ haemoglobin depletion from the host at the ring and trophozoite stages, respectively $[17,23]$. Using the estimated values of the IRBCs' $\mathrm{MCH}$ (Table 3) and haemozoin amount accumulated by parasites (Table 2), total iron of IRBCs was computed and expressed it as $\mathrm{MCH}_{\mathrm{I}}$ (Table 3). These values were in good agreement with the $\mathrm{MCH}$ values of non-infected RBCs indicating no net loss of iron from
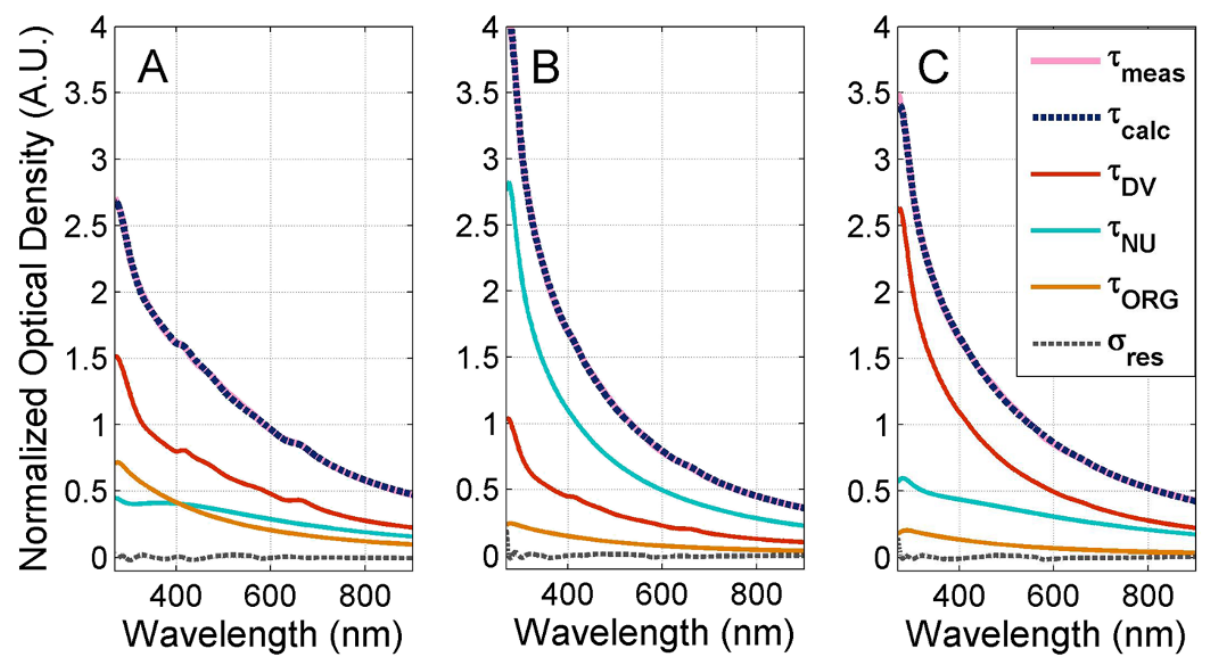

Figure 3 Interpretation of the spectra of $P$. falciparum parasites extracted from IRBCs at 15-17 hrs post treatment: (A) controls, (B) DHA treated, (C) MFQ treated. The measured spectra ( $\left.\tau_{\text {meas }}\right)$, corresponding reconstructed spectra ( $\tau_{\text {calc }}$ ), and predicted spectral contribution from the nucleus $\left(\tau_{\mathrm{NU}}\right)$, organelles $\left(\tau_{\mathrm{ORG}}\right)$, and digestive vacuole $\left(\tau_{\mathrm{DV}}\right)$ structural components are compared in each subplot. $\sigma_{\text {res }}$ denotes the difference between the measured and predicted spectra. 
IRBCs. No haemoglobin leakage from IRBCs despite parasite activity has been previously suggested [23].

\section{Mefloquine studies}

The sensitivity to the drug treatment at 10-11 hrs could be appreciated when the spectra of MFQ and DHA treated IRBCs are compared to those of the controls (Figure 4). The spectral changes became even more pronounced at the subsequent 15-17 hrs measurements (Figure 4). The structural and compositional parameters of MFQ treated parasites obtained with the interpretation model are summarized in Table 4. The structural and compositional parameters of IRBCs are given in Table 5. Comparison between the estimated parameters of control (Table 2) and MFQ treated (Table 4) parasites reveals that both treated and control parasites synchronously grew in size and no significant differences in the cell volumes between the two can be noted at any measurement time point. However, the DV volume values of MFQ treated parasites, 6.7-8.5 $\mu^{3}$ (Table 4), were nearly two-fold larger than those of controls, 3.9-6.0 $\mu^{3}$ (Table 2). The swelling of the parasite's DV has been reported as one of the MFQ effects on the parasite morphology [34]. Remarkably, cellular haemozoin levels for MFQ treated parasites, 0.03-0.04 pg (Table 4), were substantially reduced compared to that of controls, 0.15-0.28 pg (Table 2). This difference became more pronounced in the 15-22 hrs measuments. Although nucleus volume appeared to be unaffected by MFQ treatment, the estimated amount of total nucleotides per cell for MFQ treated parasites was only about a half of that for the controls (Tables 2 and 4 ).

Although the cell size of MFQ treated parasites increased between the 10-11 and 15-22 hrs measurements, lack of continuous haemozoin production and lower total nucleotide levels suggest reduced metabolic activity compared to that of the controls. The differences in these values between control and MFQ treated parasites are already apparent at 10-11 hrs measurements (Tables 2 and 4). A comparison
Table 4 Structural and compositional parameters of $P$. falciparum obtained through interpretation of the measured UV-visible-NIR spectra of MFQ treated IRBCs and parasites extracted from IRBCs

\begin{tabular}{llll}
\hline Paramer & $\mathbf{1 0 - 1 1} \mathbf{~ h r s}$ & $\mathbf{1 5 - 1 7} \mathbf{~ h r s}$ & $\mathbf{2 2} \mathbf{~ h r s}$ \\
\hline Average cell volume (fl) & $15.6 \pm 0.3$ & $21.7 \pm 0.9$ & $20.4 \pm 1.6$ \\
DV volume (fl) & $6.7 \pm 0.3$ & $7.6 \pm 0.3$ & $8.5 \pm 0.9$ \\
NU volume (fl) & $2.1 \pm 0.1$ & $2.2 \pm 0.4$ & $3.4 \pm 0.2$ \\
HZ (pg/cell) & $0.03 \pm 0.01$ & $0.03 \pm 0.01$ & $0.04 \pm 0.01$ \\
Nucleotides (fg/cell) & $26 \pm 13$ & $64 \pm 3$ & $84 \pm 11$ \\
\hline
\end{tabular}

between the measured spectra of control and MFQ treated parasites also reveals the expected absence of a haemozoin peak at $650 \mathrm{~nm}$ [15] (Figure 3). The greater contribution of the DV spectral features to the reconstructed spectrum of MFQ treated parasites was also apparent in Figure 3.

Since MFQ treated parasites stopped producing haemozoin, the host IRBCs were not as depleted of their haemoglobin content as the IRBC controls. For MFQ treated IRBCs haemoglobin depletion was only $3 \%$ at all measurement time points. Even though MFQ treated parasites did not show haemozoin production, the size of MFQ treated IRBCs continued to increase with parasite growth as that of control IRBCs. Yet, the volume gained by MFQ treated IRBCs, i.e. the difference between the IRBC volume and non-infected RBC volume, was less than the parasite volume at any given time point. For instance, at 10-11 hrs the volume increase was $2 \mu^{3}$ whereas the parasite volume was $16 \mu \mathrm{m}^{3}$ and at $22 \mathrm{hrs}$ the volume increase was $14 \mu^{3}$ whereas the parasite volume was $20 \mu^{3}$. In non-treated cultures, the parasites would have removed the excessive haemoglobin to reduce the IRBC volume gain and, therefore, to support the IRBC osmotic balance and integrity and to prevent the IRBC rupture. The results from MFQ treatment suggested that the integrity of MFQ treated IRBCs was still maintained by the parasites. They could have done it, for

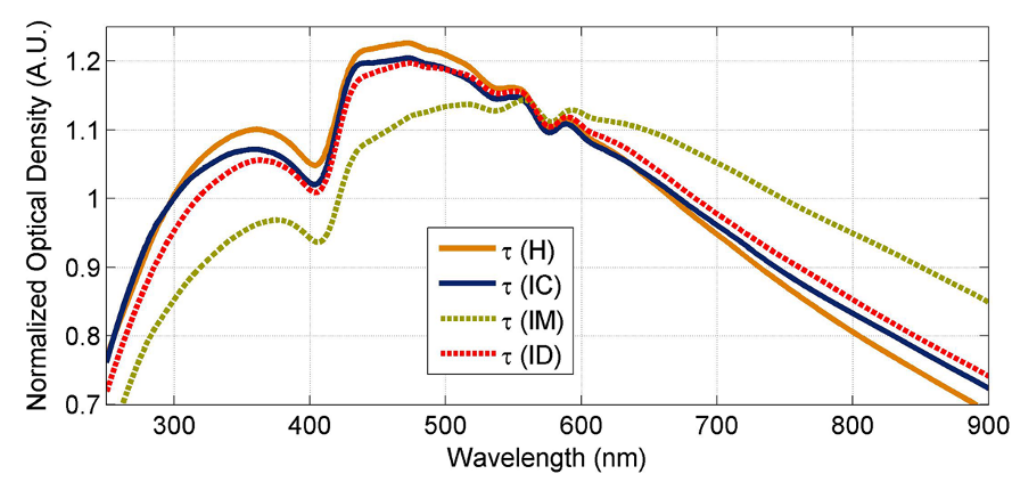

Figure 4 Reconstructed spectra of non-infected healthy RBCs (H), control IRBCs (IC), MFQ treated IRBCs (IM), and DHA treated IRBCs (ID) at 15-17 hrs post-reatment. 
Table 5 Structural and compositional parameters of IRBCs obtained through interpretation of the measured UV-visible-NIR spectra of MFQ treated cultures

\begin{tabular}{|c|c|c|c|}
\hline Parameter & $10-11 \mathrm{hrs}$ & $15-17 \mathrm{hrs}$ & $22 \mathrm{hrs}$ \\
\hline RBC volume $\left(\mu m^{3}\right)$ & $85.0 \pm 0.9$ & $85.1 \pm 1.2$ & $97.3 \pm 1.0$ \\
\hline RBC length $(\mu \mathrm{m})$ & $8.41 \pm 0.07$ & $8.31 \pm 0.02$ & $8.15 \pm 0.01$ \\
\hline RBC width $(\mu \mathrm{m})$ & $2.30 \pm 0.4$ & $2.36 \pm 0.04$ & $2.88 \pm 0.04$ \\
\hline $\mathrm{MCHC}\left(\mathrm{g} \mathrm{m}^{-1}\right)$ & $35.0 \pm 1.0$ & $38.0 \pm 0.1$ & $32.0 \pm 0.1$ \\
\hline MCH $\left(\right.$ pg cell $\left.{ }^{-1}\right)$ & $24.4 \pm 0.4$ & $24.0 \pm 0.5$ & $24.4 \pm 0.5$ \\
\hline$M C H_{1}\left(p g\right.$ cell $\left.^{-1}\right)$ & $25.2 \pm 0.2$ & $24.7 \pm 0.4$ & $25.5 \pm 0.3$ \\
\hline
\end{tabular}

instance, by removing the excessive water from the host IRBC cytosol. This suggestion is supported by the higher water to haemoglobin removal ratio calculated for treated IRBCs ( 4) compared to that for non-treated IRBCs $(\sim 2)$. The spectra of MFQ treated IRBCs differred dramatically from those of control IRBCs at all measurement time points (Figures 2 and 3). The dominant cause of this distinction was the greater refractive index values of MFQ treated IRBC which have a greater haemoglobin content compared to that of non-treated IRBCs.

\section{Dihydroartemisinin studies}

It has been reported that oxidative stress caused by DHA actually kills malaria parasites inside IRBCs [35]. Here we examine what it meant in terms of the changes in the structure and composition of parasites and the effects of those changes on the spectral features of DHA treated IRBCs. The structural and compositional parameters of DHA treated parasites obtained through interpretation of the spectra measured at $10-11$ and $15-17$ hrs are summarized in Table 6. The structural and compositional parameters of DHA treated IRBCs are given in Table 7. All parameters estimated for DHA treated parasites were already different from those of the controls at 10-11 hrs post treatment. The cell size and organelle volumes were about 30\% lower for DHA treated parasites than those for the controls. Similarly to MFQ treated parasites, DHA treated parasites did not accumulate haemozoin. The total

Table 6 Structural and compositional parameters of $P$. falciparum obtained through interpretation of the measured UV-visible-NIR spectra of DHA treated IRBCs and parasites extracted from IRBCs

\begin{tabular}{lll}
\hline Paramer & $\mathbf{1 0 - 1 1} \mathbf{~ h r s}$ & $\mathbf{1 5 - 1 7} \mathbf{~ h r s}$ \\
\hline Average cell volume (fl) & $10.5 \pm 1.3$ & $10.2 \pm 2.1$ \\
DV volume (fl) & $2.9 \pm 0.3$ & $3.0 \pm 0.3$ \\
$\mathrm{NU}$ volume (fl) & $1.8 \pm 0.3$ & $2.0 \pm 0.8$ \\
$\mathrm{HZ}$ (pg/cell) & $0.05 \pm 0.03$ & $0.04 \pm 0.02$ \\
Nucleotides in NU (fg/cell) & $15 \pm 13$ & $14 \pm 2$ \\
\hline
\end{tabular}

Table 7 Structural and compositional parameters of IRBCs obtained through interpretation of the measured UV-visible-NIR spectra of DHA treated cultures

\begin{tabular}{lll}
\hline Parmeter & $\mathbf{1 0 - 1 1} \mathbf{~ h r s}$ & $\mathbf{1 5 - 1 7} \mathbf{~ h r s}$ \\
\hline RBC volume $\left(\mu \mathrm{m}^{3}\right)$ & $82.9 \pm 0.7$ & $89.1 \pm 2.2$ \\
RBC length $(\mu \mathrm{m})$ & $8.37 \pm 0.02$ & $8.26 \pm 0.07$ \\
RBC width $(\mu \mathrm{m})$ & $2.27 \pm 0.02$ & $2.41 \pm 0.09$ \\
$\mathrm{MCHC}\left(\mathrm{g} \mathrm{ml}^{-1}\right)$ & $32.6 \pm 0.55$ & $30.4 \pm 0.9$ \\
$M C H\left(p g\right.$ cell $\left.^{-1}\right)$ & $23.6 \pm 0.5$ & $24.1 \pm 0.4$ \\
$\mathrm{MCH}_{\text {। }}\left(\mathrm{pg} \mathrm{cell} \mathrm{l}^{-1}\right)$ & $25.0 \pm 0.5$ & $25.3 \pm 0.4$ \\
\hline
\end{tabular}

nucleotide values for DHA treated parasites were considerably lower than those for the controls and MFQ treated parasites. The differences were already dramatic at 10-11 hrs and increased at the next measurement time point. All parameter values estimated for DHA treated parasites remained fairly unchanged between the 10-11 and 15-17 hrs measurements. This consistency suggests no parasite growth between measurements; therefore, DHA treated parasites were not alive by the 10-11 hrs measurement and the structural and compositional characteristics captured reflected those of dead cells. The spectral features of DHA treated parasites were distinguished from those of the controls and MFQ treated parasites by the steeper slope indicative of the smaller cell size and the reduced peak at $650 \mathrm{~nm}$ indicative of the smaller haemozoin content (Figure 3).

Similarly to MFQ treatment, haemoglobin depletion of DHA treated IRBCs was significantly reduced compared to that of IRBC controls. DHA treated IRBCs were depleted by only $5 \%$ in haemoglobin content at $10-11 \mathrm{hrs}$ and remained such at 15-17 hrs. The spectral interpretation analysis showed that DHA treated IRBCs increased in volume from the 10-11 hrs to 15-17 hrs measurements (Table 7). Since the parasite size did not increase between the measurements and haemoglobin content remained the same, this volume gain could be attributed to the influx of water into DHA treated IRBCs. This allowed DHA treated IRBCs to regain their initial MCHC value that was disturbed by the parasite invasion (Table 7). Such measure resulted in the reduced refractive index values of DHA treated IRBCs compared to MFQ treated IRBCs. The refractive index of the cells has a pronounced influence on the spectral features. The refractive indices of control IRBCs and DHA treated IRBCs were practically the same due to similar MCHC values at 10-11 hrs measurement (Tables 3 and 7). In addition, given the close sizes of IRBCs and parasites between controls and DHA treated cultures, the spectral features of control and DHA treated IRBCs overlapped above $400 \mathrm{~nm}$ at 10-11 hrs post treatment (Figure 2). However, subsequent changes in the refractive index of control IRBCs attributable to the parasite growth 
and haemoglobin depletion led to greater discrimination between the spectral features of control and DHA treated IRBCs at 15-17 hrs measurement (Figure 4).

\section{Conclusion}

A comprehensive spectral interpretation model was developed in order to extract information on the structure and composition of the intraerythrocytic parasite and its host from spectroscopic measurements. Quantitative analysis of the UV-visible spectra of IRBCs parasitized with the most lethal malaria parasite P. falciparum allowed insights into the parasite and host interactions. These results appeared to be robust when compared to the reported measured values and estimates made with other methods.

Notable changes occur in the IRBC spectral features due to reshaping of the infected cells, growth of intracellular parasites, and depletion of haemoglobin from the host IRBC cytosol. The spectral interpretation analysis indicated a mass balance between the total iron in the IRBCs (haemoglobin in cytosol plus haemozoin in parasites) and that in healthy RBCs (haemoglobin) indicating no net loss of iron from IRBCs despite a parasite-induced increase in the permeability of the IRBC membrane. IRBCs increased in volume and became more spherical with parasite's progression from the ring stage into trophozoite stage. Because of the parasite's effort to maintain the osmotic balance and integrity of the host IRBC, the IRBC volume was less than a sum of those of a healthy RBC and a parasite at trophozoite stage. This effort included excessive uptake and break up of haemoglobin [36] followed by the conversion of haem into haemozoin pigment and removal of free amino acids and other protein pieces [18,21].

Since spectroscopic measurements have high sensitivity to the size and composition of particles and cells, the action of anti-malarial drugs on the structure and composition of the parasites and IRBCs led to distinguishing spectral features. Our results demonstrated that the effects of MFQ and DHA could readily be appreciated at 10-11 hrs after drug administration. Specifically, MFQ blocked haemozoin formation and led to the swelling of the parasite's digestive vacuole. Since the host IRBC was minimally depleted in haemoglobin, it increased in volume and changed shape with parasite's growth. On the other hand, as DHA terminated the parasite's metabolic activity, and it reduced the sizes of the parasite's cell body and organelles. The cell and organelle volumes of DHA treated parasites were about $60-70 \%$ of those of nontreated parasites grown in parallel as controls. Haemoglobin depletion from the host cytosol and haemozoin accumulation discontinued when DHA treatment became effective. These findings suggested that a spectral interpretation analysis could have significant potential for drug effect evaluation of parasite isolates or for therapeutic monitoring of malaria patients.

\section{Competing interests}

The authors declare that they have no competing interests.

\section{Authors' contributions}

YS performed the spectroscopic measurements, conducted data analysis, and drafted the manuscript. JP carried out parasite cultivation and treatment. JP and WM conceived and designed the culture experiments. LGR, DH, and JS contributed to the data interpretation and manuscript preparation. All authors had full access to all data in the study, read and approved the manuscript.

\section{Acknowledgment}

This work was supported by the Florida High Tech Corridor 09-08 Industry Seed grant (6408-1024-00) and by Claro Scientific LLC. We thank Dr. Kyle for training in culture preparation and help with the design of experiments.

Received: 4 December 2012 Accepted: 2 August 2013

Published: 30 August 2013

\section{References}

1. Campuzano-Zuluaga G, Hänscheid T, Grobusch MP: Automated haematology analysis to diagnose malaria. Malar J 2010, 9:346.

2. WHO: World Malaria Report. Geneva, Switzerland: World Health Organization Press; 2008. 2008.

3. Moges B, Amare B, Belyhun Y, Tekeste Z, Gizachew M, Workineh M, Gebrehiwot A, Woldeyohannes D, Mulu A, Kassu A: Comparison of CareStart ${ }^{\mathrm{TM}} \mathrm{HRP} 2 / \mathrm{pLDH}$ COMBO rapid malaria test with light microscopy in north-west Ethiopia. Malar J 2012, 11:234.

4. Santana-Morales MA, Afonso-Lehmann RN, Quispe MA, Reyes F, Berzosa P, Benito A, Valladares B, Martinez-Carretero E: Microscopy and molecular biology for the diagnosis and evaluation of malaria in a hospital in a rural area of Ethiopia. Malar J 2012, 11:199.

5. Prescott WR, Jordan RG, Grobusch MP, Chinchilli VM, Kleinschmidt I, Borovsky J, Plaskow M, Torrez M, Mico M, Schwabe C: Performance of a malaria microscopy image analysis slide reading device. Malar J 2012, 11:155.

6. Marloes Heutmekers M, Philippe Gillet P, Maltha J, Scheirlinck A, Cnops L, Bottieau E, Esbroeck MV, Jacobs J: Evaluation of the rapid diagnostic test CareStart pLDH Malaria (Pf-pLDH/pan-pLDH) for the diagnosis of malaria in a reference setting. Malar J 2012, 11:204.

7. Fontecha GA, Mendoza M, Banegas E, Poorak M, De Oliveira AM, Mancero T, Udhayakumar V, Lucchi NW, Mejia RE: Comparison of molecular tests for the diagnosis of malaria in Honduras. Malar J 2012, 11:119.

8. Lamikanra AA, Dobaño C, Jiménez A, Nhabomba A, Tsang HP, Guinovart C, Manaca MN, Quinto L, Aguilar R, Cisteró P, Alonso PL, Roberts DJ, Mayor A: A direct comparison of real time PCR on plasma and blood to detect Plasmodium falciparum infection in children. Malar J 2012, 11:201.

9. Noedl H, Wongsrichanalai C, Wernsdorfer WH: Malaria drug-sensitivity testing: new assays new perspectives. Trends Parasitol 2003, 19:175-181.

10. Marfurt J, Smith TA, Hastings IM, Mueller I, Sie A, Oa O, Baisor M, Reeder JC, Beck HP, Genton B: Plasmodium falciparum resistance to anti-malarial drugs in Papua New Guinea: evaluation of a community-based approach for the molecular monitoring of resistance. Malar J 2010, 9:8.

11. Menard S, Morlais I, Tahar R, Sayang C, Mayengue PI, Iriart X, Benoit-Vical F, Lemen B, Magnaval JF, Awono-Ambene P, Basco LK, Berry A: Molecular monitoring of Plasmodium falciparum drug susceptibility at the time of the introduction of artemisinin-based combination therapy in Yaoundé, Cameroon: Implications for the future. Malar J 2012, 11:113.

12. Marfurt J, Mueller I, Sie A, Maku P, Goroti M, Reeder JC, Beck HP, Genton B: Low efficacy of amodiaquine or chloroquine plus sulfadoxine-pyrimethamine against Plasmodium falciparum and $P$. vivax malaria in Papua New Guinea. Am J Trop Med Hyg 2007, 77:947-954.

13. Frita R, Rebelo M, Pamplona A, Vigario AM, Mota MM, Grobusch MP, Hänscheid T: Simple flow cytometric detection of haemozoin containing leukocytes and erythrocytes for research on diagnosis, immunology and drug sensitivity testing. Malar J 2011, 10:74.

14. Serebrennikova YM, Patel J, García-Rubio LH: Interpretation of the UV-visible spectra of malaria parasite Plasmodium falciparum. Appl Opt 2010, 49:180-188.

15. Bannister LH, Hopkins JM, Fowler RE, Krishna S, Mitchell GH: A brief illustrated guide to the ultrastructure of Plasmodium falciparum asexual blood stages. Parasitol Today 2000, 16:427-433. 
16. Magowan C, Brown JT, Liang J, Heck J, Coppel RL, Mohandas N, Meyer-Ilse W: Intracellular structures of normal and aberrant Plasmodium falciparum malaria parasites imaged by soft x-ray microscopy. Proc Natl Acad Sci USA 1997, 94:6222-6227.

17. Park YK, Diez-Silva M, Popescu G, Lykotrafitis G, Choi W, Feld MS, Suresh S: Refractive index maps and membrane dynamics of human red blood cells parasitized by Plasmodium falciparum. Proc Natl Acad Sci USA 2008, 105:13730-13735.

18. Kirk K: Membrane transport in the malaria-infected erythrocyte. Physiol Rev 2001, 81:495-537.

19. Glushakova S, Yin D, Gartner N, Zimmerberg J: Quantification of malaria parasite release from infected erythrocytes: inhibition by protein-free media. Malar J 2007, 6:61.

20. Gruenberg J, Allred DR, Sherman IW: Scanning electron microscope-analysis of the protrusions (knobs) present on the surface Plasmodium falciparum-infected erythrocytes. J Cell Biol 1983, 97:795-802.

21. Haldar K, Mohandas N: Erythrocyte remodeling by malaria parasites. Curr Opin Hematol 2007, 14:203-209.

22. Francis SE, Sullivan DJ, Goldberg DE: Hemoglobin metabolism in the malaria parasite Plasmodium falciparum. Ann Rev Microbiol 1997, 51:97-123.

23. Egan TJ, Combrinck JM, Egan J, Hearne GR, Marques HM, Ntenteni S, Sewell BT, Smith PJ, Taylor D, van Schalkwyk DA, Walden JC: Fate of haem iron in the malaria parasite Plasmodium falciparum. Biochem J 2002, 365:343-347.

24. Moore LR, Fujioka H, Williams PS, Chalmers JJ, Grimberg B, Zimmerman PA: Hemoglobin degradation in malaria-infected erythrocytes determined from live cell magnetophoresis. FASEB J 2006, 20:747-749.

25. Serebrennikova YM, Patel J, Milhous WK, García-Rubio LH: Quantitative analysis of morphological alterations in Plasmodium falciparum infected red blood cells through theoretical interpretation of spectral measurements. J Theoret Biol 2010, 265:493-500.

26. Trager W, Jensen JB: Cultivation of malarial parasites. Nature 1978, 273:621-622.

27. Serebrennikova YM, García-Rubio LH: Modeling and interpretation of the extinction spectra of oriented non-spherical composite particles: application to biological cells. Appl Opt 2010, 49:4460-4471.

28. Tarasov P, Yurkin M, Avrorov P, Semyanov K, Hoekstra A, Maltsev V, NATO Sci. Series II: Math Physics Chemistry: Optics of erythrocytes. In Optics of Biological Particles, Volume 238. Edited by Hoekstra A, Maltsev V, Videen G. Netherlands: Springer; 2007:243-259.

29. Alupoaei CE, García Rubio LH: An interpretation model for the UV-vis spectra of microorganisms. Chem Eng Comm 2005, 192:198-218.

30. Latimer P: Light scattering by ellipsoids. J Coll Inter Sci 1975, 53:102-109.

31. Gardner MJ, Hall N, Fung E, White O, Berriman M, Hyman RW, Carlton JM, Pain A, Nelson KE, Bowman S, Paulsen IT, James K, Eisen JA, Rutherford K, Salzberg SL, Craig A, Kyes S, Chan MS, Nene V, Shallom SJ, Suh B, Peterson J, Angiuoli S, Pertea M, Allen J, Selengut J, Haft D, Mather MW, Vaidya AB, Martin DMA, et al: Genome sequence of the human malaria parasite Plasmodium falciparum. Nature 2002, 419:498-511.

32. Nash GB, O'Brien E, Gordon-Smith EC, Dormandy JA: Abnormalities in the mechanical properties of red blood cells caused by Plasmodium falciparum. Blood 1989, 74:855-861.

33. Shi H, Li A, Yin J, Tan KSW, Lim CT: AFM study of the cytoskeletal structures of malaria infected erythrocytes. Proc ICBME 2009, 23:1965-1968.

34. Jacobs GH, Aikawa M, Milhous WK, Rabbege JR: An ultrastructural study of the effects of mefloquine on malaria parasites. Am J Trop Med Hyg 1987, 36:9-14.

35. Zhang J, Krugliak M, Ginsburg H: The fate of ferriprotorphyrin IX in malaria infected erythrocytes in conjunction with the mode of action of antimalarial drugs. Mol Biochem Parasitol 1999, 99:129-141.

36. Krugliak M, Zhang J, Ginsburg H: Intraerythrocytic Plasmodium falciparum utilizes only a fraction of the amino acids derived from the digestion of host cell cytosol for the biosynthesis of its proteins. Mol Biochem Parasitol 2002, 119:249-256.

doi:10.1186/1475-2875-12-305

Cite this article as: Serebrennikova et al: Spectrophotometric detection

of susceptibility to anti-malarial drugs. Malaria Journal 2013 12:305.

\section{Submit your next manuscript to BioMed Central and take full advantage of:}

- Convenient online submission

- Thorough peer review

- No space constraints or color figure charges

- Immediate publication on acceptance

- Inclusion in PubMed, CAS, Scopus and Google Scholar

- Research which is freely available for redistribution

Submit your manuscript at www.biomedcentral.com/submit
Biomed Central 